BMJ

Open

Gastroenterology

\title{
Low yield of head CT in cirrhotic patients presenting with hepatic encephalopathy
}

\author{
Angy Hanna, ${ }^{1}$ Inayat Gill, ${ }^{1}$ Zaid Imam (1) ,, Alexandra Halalau, ${ }^{3,4}$ Laith $\mathrm{H}$ Jamil ${ }^{2,4}$
}

To cite: Hanna A, Gill I, Imam Z, et al. Low yield of head CT in cirrhotic patients presenting with hepatic encephalopathy. BMJ Open Gastro

2021;8:e000609. doi:10.1136/ bmjgast-2021-000609

Received 21 January 2021

Revised 18 April 2021

Accepted 19 April 2021
Check for updates

(c) Author(s) (or their employer(s)) 2021. Re-use permitted under CC BY-NC. No commercial re-use. See rights and permissions. Published by BMJ.

${ }^{1}$ Internal Medicine, William Beaumont Hospital, Royal Oak, Michigan, USA

${ }^{2}$ Gastroenterology and Hepatology, William Beaumont Hospital, Royal Oak, Michigan, USA

${ }^{3}$ Internal Medicine, Oakland University William Beaumont School of Medicine, Rochester, Michigan, USA

${ }^{4}$ Oakland University William Beaumont School of Medicine, Rochester, Michigan, USA

Correspondence to Dr Angy Hanna; angy.hanna@beaumont.org

\section{ABSTRACT}

Goals and background The utility of routine head CT (HCT) in hepatic encephalopathy (HE) evaluation is unclear. We investigated HCT yield in detecting acute intracranial abnormalities in cirrhotic patients presenting with HE. Study Retrospective review of cirrhotic patient encounters with HE between 2016 and 2018 at Beaumont Health, in Michigan was performed. A low-risk (LR) indication for HCT was defined as altered mental status (AMS), which included dizziness and generalised weakness. A high-risk (HR) indication was defined as trauma/fall, syncope, focal neurological deficits (FNDs) or headache. Descriptive statistics and univariate/multivariate analyses by logistic regression were performed using SPSS to identify HCT abnormality correlates.

Results Five hundred twenty unique encounters were reviewed. Mean age was 63.4 (12.1) years, 162 (37.5\%) had alcoholic cirrhosis and median Model for EndStage Liver Disease (MELD)-score was 17 (13-23). LR indication was reported in 408 (78.5\%) patients and FNDs reported in $24(4.6 \%)$ patients. Only $13(2.5 \%)$ patients were found to have an acute intracranial pathology (seven haemorrhagic stroke, two ischaemic stroke, four subdural haematoma). Aspirin use prior to presentation (aOR 4.6, 95\% $\mathrm{Cl} 1.1$ to 19.2), and HR indication (aOR 7.3, $95 \% \mathrm{Cl} 2.3$ to 23.8) were independent correlates of acute intracranial pathology on HCT. Age, sex, MELD-score, haemoglobin, platelet count, race and cirrhosis aetiology did not correlate with HCT abnormalities. Number needed to screen to identify one acute pathology was 14 in HR indications versus 82 for LR indications.

Conclusion Routine HCTs in cirrhotic patients presenting with HE with AMS in the absence of history of trauma, headache, syncope, FNDs or aspirin use is of low diagnostic yield.

\section{INTRODUCTION}

Hepatic encephalopathy (HE) is a significant complication that occurs in 30\%-45\% of patients with portal hypertension and cirrhosis. ${ }^{1}$ It manifests as a wide spectrum of changes in consciousness, personality, motor function and/or cognition. ${ }^{2}$ Pathophysiology of $\mathrm{HE}$ is complex and involves dysregulation between portosystemic shunting, excess ammonia, inflammatory mediators, oxidative stress and cerebral edema. ${ }^{3}$ There are no pathognomonic clinical or imaging findings
Summary

What is already known about this subject?

- Routine head CT in cirrhotic patients presenting with hepatic encephalopathy and altered mental status in the absence of focal neurological deficits is low yield for detecting intracranial haemorrhage.

What are the new findings?

- The practice of routine head CT in cirrhotic patients presenting with altered mental status in the $a b$ sence of a history of trauma or focal neurological deficits is of low yield to detect not only intracranial haemorrhage but any cerebral structural abnormalities. Additionally, rates of intracranial haemorrhage and ischaemic strokes in patients presenting with hepatic encephalopathy is low and is not affected by liver disease severity or cirrhosis aetiology.

How might it impact on clinical practice in the foreseeable future?

- Reserving the use of head CT in cirrhotic patients presenting to the emergency room with altered mental status to only when a history of trauma or focal neurological deficits is present.

that specifically arise in patients with $\mathrm{HE}$, and thus detailed evaluation of alternative aetiologies of brain dysfunction is needed prior to diagnosis. ${ }^{24}$

Initial workup in a patient with suspected HE includes a thorough physical examination and laboratory testing to evaluate for any evidence of bleeding, electrolyte abnormalities or infection. ${ }^{4}$ Intracranial haemorrhage (ICH) is a feared cause for altered mentation in cirrhotic patients. However, ICH is typically accompanied by focal neurological deficit(s) (FND) on physical examination. Despite low diagnostic yield in the absence of FND demonstrated by several studies, ${ }^{5-7}$ noncontrast head CT (HCT) is still frequently used in evaluation of cirrhotic patients presenting with altered consciousness, contributing to higher healthcare costs. ${ }^{5}$ The American Association for the Study of Liver Diseases (AASLD) and European 
Association for the Study of the Liver guidelines do not offer concrete recommendations on the role of imaging in HE, with the only data available on the topic in the AASLD guidelines is citing a fivefold increased relative risk of ICH in cirrhotic patients ${ }^{8}$ and common practice patterns where neuroimaging is routinely obtained in patients presenting with HE. ${ }^{49}$

This study aims to investigate the yield of obtaining routine HCTs in cirrhotic patients presenting with HE and investigating correlates of acute intracranial abnormalities on imaging in this population.

\section{METHODOLOGY}

Population

William Beaumont Hospital, Royal Oak (WBH-RO) is a tertiary care referral centre in Michigan, USA with an active liver transplant programme since $2010 .{ }^{6} \mathrm{~A}$ retrospective cohort of emergency department (ED) and hospitalised adult patient encounters at WBH-RO presenting with HE between 1 January 2016 and 31 December 2018 was identified using an electronic health record (EHR) query of the following International Classification of Diseases (ICD) codes: (1) $572.2 \mathrm{HE}$ (ninth edition) and (2) K72.91 Hepatic failure, unspecified with coma (10th edition). Patients were included if they had a HCT without intravenous contrast on presentation or during the hospitalisation, and a diagnosis of liver cirrhosis. Both criteria were confirmed by manual chart review. If multiple HCTs were performed, only the initial HCT findings were analysed. Exclusion criteria included patients with prior known HCT abnormalities prior to the encounter such as encephalomalacia, abnormalities secondary to prior strokes or space occupying lesions.

\section{VARIABLES}

Variables were abstracted using automated reports of WBH-RO's EHRs generated by ToadDataPoint multiplatform database query tool (EPIC System, Verona, Wisconsin, USA). Variables included patient demographics, medical comorbidities; laboratory values; aspirin, $\mathrm{P}_{2} \mathrm{Y}_{12}$ and anticoagulant use; aetiology of cirrhosis; and HCT reports for findings. Records were manually and independently reviewed by two reviewers (AH and IG) for presence of new FNDs and for HCT indications reported in physician notes during the ED/ hospital encounter. Any discrepancies were settled by discussion with a third reviewer (ZI).

FNDs included focal weakness, paraesthesias, dysarthria, ataxia and seizures. Altered mental status (AMS) was defined as altered level of consciousness, change in cognition, disorientation or behavioural change from known baseline reported by collateral or known to providers from prior encounters as defined by Rahimi and Rockey, ${ }^{7}$ and included additional complaints of generalised weakness and dizziness if present. Model for End-Stage Liver Disease (MELD) scores were calculated for each encounter using predefined algorithms. ${ }^{7}$

\section{OUTCOMES}

The primary outcome evaluated was the frequency of acute intracranial abnormalities on index HCTs performed in cirrhotic adult patients presenting with HE. Acute intracranial abnormalities were defined as the presence of one or more of: (1) acute ischaemic stroke, (2) acute haemorrhagic stroke, (3) subdural/epidural haemorrhage or (4) space-occupying lesion(s) as defined by the reading radiologist. The presence of acute HCT findings was confirmed on MRI if it was obtained in the same admission and replaced with the MRI findings if different. Cirrhotic patients with HE presenting with AMS in the absence of FNDs or trauma have been reported to have low risks of ICH. ${ }^{5}$ We, therefore, classified AMS as a low-risk indication for HCT. Other indications such as FNDs, trauma, falls, syncope or headache were classified as high-risk indications for HCT. Additionally, HCT indications were stratified into five separate categories: (1) presence of FND, (2) AMS, (3) Fall, trauma and syncope, (4) headache and (5) other categories. Finally, a simple cost analysis for HCT use in low-risk patients was reported.

\section{STATISTICAL ANALYSIS}

Parametric continuous variables were reported as means and SD and non-parametric continuous variables as medians and IQR. Categorical variables were reported as frequencies and proportions. Student's t-tests and kruskal wallis tests were performed to compare parametric and non-parametric continuous variables, respectively. $\chi^{2}$ tests were performed to compare categorical variables. Univariate and multivariate stepwise logistic regression analyses were performed to identify clinical variables associated with acute intracranial abnormalities on HCT. MELD scores were used in univariate analyses as surrogates for their constituents (international normalised ratio, total bilirubin and serum creatinine). Multivariate analysis was performed for only statistically significant variables to minimise overfitting. $\mathrm{P}<0.05$ demonstrated statistical significance in all analyses. All analyses were performed using IBM SPSS Statistics for Windows, V.23.0 (IBM).

\section{RESULTS}

\section{Cohort demographics and clinical characteristics}

A total of 520 unique encounters (432 unique patients) were eligible for inclusion. The most common aetiology of cirrhosis was alcoholic liver disease in 162 (37.5\%) patients, followed by metabolic causes (non-alcoholic steatohepatitis, haemochromatosis and Wilson's disease) in $87(20.1 \%)$ patients, chronic viral hepatitis B and C in $53(12.3 \%)$, a combination of alcoholic liver disease and another aetiology in 22 patients $(5.1 \%)$ and autoimmune liver disease (autoimmune hepatitis, primary biliary cirrhosis, primary sclerosing cholangitis) in 11 patients $(2.5 \%)$. Aetiology of cirrhosis was cryptogenic 
Table 1 Cohort demographics and clinical characteristics

\begin{tabular}{lc}
\hline Patient characteristics (No=432) & No (\%) \\
\hline General characteristics & $63.4(12.1)$ \\
\hline Mean age (SD) & $246(56.9)$ \\
\hline Male & $324(75.0)$ \\
\hline Caucasian race & $162(37.5)$ \\
\hline Cirrhosis aetiology & $97(22.5)$ \\
\hline Alcoholic cirrhosis & $87(20.1)$ \\
\hline Cryptogenic/unknown & \\
\hline $\begin{array}{l}\text { Metabolic (NASH, haemochromatosis, } \\
\text { Wilson's) }\end{array}$ & $11(2.5)$ \\
Viral hepatitis B and C & $22(5.1)$ \\
\hline $\begin{array}{l}\text { Autoimmune (AlH, PBC, PSC) } \\
\text { Combination of alcoholic liver disease } \\
\text { and other aetiology }\end{array}$
\end{tabular}

Medical comorbidities

\begin{tabular}{lc}
\hline Atrial fibrillation & $50(11.6)$ \\
\hline Venous thromboembolic disease & $23(5.3)$ \\
\hline CVA or TIA & $26(6.0)$ \\
\hline $\begin{array}{l}\text { Coronary artery disease or peripheral } \\
\text { artery disease }\end{array}$ & $89(20.6)$ \\
\hline Encounter characteristics (no=520) & \\
\hline Laboratory values & Median (IQR) \\
\hline WCC (x 10 $/$ L) & $6.3(4.4-9.6)$ \\
\hline Platelet count (x109/L) & $116(77-174)$ \\
\hline MELD score (n=473) & $17(13-23)$ \\
\hline Haemoglobin (g/dL) & $10.8(9.1-12.6)$ \\
\hline Serum creatinine (mg/dL) & $1.3(0.9-2.1)$ \\
\hline International normalised ratio & $1.4(1.2-1.8)$ \\
\hline Total bilirubin (mg/dL) & $2(1.1-3.7)$ \\
\hline Medications & No (\%) \\
\hline $\begin{array}{l}\text { Anticoagulant use (direct oral } \\
\text { anticoagulants/warfarin) }\end{array}$ & $67(12.9)$ \\
\hline Aspirin use & $196(37.7)$ \\
\hline $\begin{array}{l}\text { Other antiplatelet agent (ticagrelor, } \\
\text { prasugrel, clopidogrel) }\end{array}$ & $61(11.7)$ \\
\hline
\end{tabular}

.AlH, autoimmune hepatitis; MELD, model for end stage liver disease; NASH, non-alcoholic steatohepatitis; PBC, primary biliary cholangitis; PSC, primary sclerosing cholangitis; WCC, white cell count.

or unknown in $97(22.5 \%)$ patients. Table 1 summarises cohort demographics and clinical characteristics.

\section{HCT indications, findings and correlates with abnormal} findings

A total of $13(2.5 \%)$ acute intracranial abnormalities were identified on index HCTs. Of these, 7 (1.3\%) were haemorrhagic strokes, $2(0.4 \%)$ acute ischaemic strokes and 4 $(0.8 \%)$ subdural haematomas. No epidural haematomas or space-occupying lesions were identified. Four $(30.8 \%)$ of these patients had FNDs, three $(23.1 \%)$ patients had a
Table 2 Head CT findings and indications

\begin{tabular}{lc|}
\hline & No (\%) \\
\hline Total no of scans & $520(100)$ \\
Head CT indications & \\
\hline Low risk (altered mental status) & $408(78.5)$ \\
\hline High risk & $112(21.5)$ \\
\hline Fall, trauma or syncope & $67(12.9)$ \\
Focal neurological deficit(s) & $28(5.4)$ \\
Headache & $9(1.7)$ \\
Others & $6(1.2)$
\end{tabular}

Head CT findings

$\begin{array}{lr}\text { Acute intracranial abnormality } & 13(2.5) \\ \text { Acute ischaemic stroke } & 7(1.3) \\ \text { Haemorrhagic stroke } & 2(0.4) \\ \text { Subdural haemorrhage } & 4(0.8) \\ \text { Focal neurological deficits } & \\ \text { By type: } & 14(2.7) \\ \text { Seizure } & 6(1.2) \\ \text { Speech deficits } & 3(0.6) \\ \text { Motor deficits } & 2(0.4) \\ \text { Visual deficits } & 2(0.4) \\ \text { Combination of more than one deficit } & 1(0.2) \\ \text { Sensory deficits } & \end{array}$

history of fall/trauma, two $(15.4 \%)$ a history of syncope, one had a cardiac arrest $(7.7 \%)$ and three $(23.1 \%)$ patients had a history of AMS.

In terms of clinical presentation, 28 (5.4\%) patients presented with FNDs. The most common FND was seizure in 14 patients, followed by speech deficits in six patients, motor deficits in three patients, acute visual deficits in two patients and sensory deficits in one patient. The remaining two patients had more than one deficit reported.

Low-risk indications were present in 408 (78.5\%) encounters. Of the 112 high-risk indications, $67(12.9 \%)$ were fall, trauma or syncope, $28(5.4 \%)$ were FNDs and $9(1.7 \%)$ were headache. The remaining six encounters involved one totally unresponsive patient, one patient with cardiac arrest, one patient with conjunctival haemorrhage, and an unclear pathology in two patients. These six encounters were included as high-risk indications. Table 2 summarises HCT findings and indications.

Six $(46.1 \%)$ of the 13 patients with findings on HCTs reported aspirin use. Using univariate analysis, presence of a high-risk indication (OR 6.2, 95\% CI 2.0 to 19.3), aspirin use prior to presentation (OR 5.8, 95\% CI 1.6 to 21.2), history of atrial fibrillation (AF) (OR 3.38, 95\% CI 1.00 to 11.30$)$ and history of coronary artery disease (CAD) (OR 3.2, 95\% CI 1.03 to 10.2 ) were significantly associated with presence of acute intracranial abnormalities on HCTs. Using multivariate analysis, aspirin use 
Table 3 Univariate analysis of correlates of acute intracranial abnormalities on head CT (HCT)

\begin{tabular}{|c|c|c|}
\hline Univariate analysis & OR $(95 \% \mathrm{Cl})$ & $P$ value \\
\hline \multicolumn{3}{|l|}{ Demographics } \\
\hline Mean age & $1.0(0.9$ to 1.0$)$ & 0.581 \\
\hline Male sex & $0.2(0.1$ to 1.1$)$ & 0.059 \\
\hline Other race compared with Caucasian & $0.6(0.2$ to 2.0$)$ & 0.441 \\
\hline \multicolumn{3}{|l|}{ Medication use prior to encounter } \\
\hline Anticoagulants (direct oral anticoagulants/warfarin) & $2.1(0.6$ to 7.8$)$ & 0.277 \\
\hline Aspirin & 5.8 (1.6 to 21.2$)$ & 0.008 \\
\hline Other antiplatelet agents (ticagrelor, prasugrel, clopidogrel) & $1.4(0.3$ to 6.4$)$ & 0.680 \\
\hline \multicolumn{3}{|l|}{ Cirrhosis aetiology compared with alcoholic cirrhosis } \\
\hline Viral hepatitis B and C & 0.9 (0.3 to 3.3$)$ & 0.888 \\
\hline Combination of alcoholic liver disease and other aetiology & $0.4(0.1$ to 4.0$)$ & 0.463 \\
\hline Metabolic (NASH, haemochromatosis, Wilson's) & $-*$ & - \\
\hline Autoimmune (AlH, PBC, PSC) & $-*$ & - \\
\hline Cryptogenic/unknown & 0.5 (0.1 to 2.8$)$ & 0.435 \\
\hline \multicolumn{3}{|l|}{ Comorbidities } \\
\hline Atrial fibrillation & 3.4 (1.0 to 11.3$)$ & 0.049 \\
\hline Venous thromboembolic disease & $1.6(0.2$ to 12.9$)$ & 0.655 \\
\hline Coronary artery disease & $3.2(1.03$ to 10.2$)$ & 0.044 \\
\hline Peripheral vascular disease & $-*$ & - \\
\hline MELD score & 1.07 (1.00 to 1.2$)$ & 0.061 \\
\hline Platelet count (cells/mm³) & $1.00(1.00$ to 1.01$)$ & 0.540 \\
\hline Serum haemoglobin (g/L) & $0.93(0.74$ to 1.16$)$ & 0.508 \\
\hline \multicolumn{3}{|l|}{ By HCT indication compared with AMS (low-risk indication) } \\
\hline Presence of high-risk indication & 6.2 (2.0 to 19.3$)$ & 0.002 \\
\hline Focal neurological deficit & 6.2 (1.2 to 33.5$)$ & 0.034 \\
\hline Trauma, fall or syncope & 6.7 (1.9 to 23.9$)$ & 0.003 \\
\hline Headache & $-{ }^{*}$ & - \\
\hline Other indications & 9.0 (0.9 to 84.7$)$ & 0.056 \\
\hline
\end{tabular}

*Showed complete separation and hence unable to implement in regression analysis.

$\mathrm{AlH}$, autoimmune hepatitis; AMS, altered mental status.; MELD, model for end-stage liver disease; NASH, non-alcoholic steatohepatitis; PBC, primary biliary cholangitis; PSC, primary sclerosing cholangitis.

prior to presentation (aOR:4.6, 95\% CI 1.1 to 19.2 ), and presence of a high-risk indication (aOR 7.3, 95\% CI 2.3 to 23.8$)$, but not $\mathrm{CAD}(\mathrm{p}=0.367)$ or $\mathrm{AF}(\mathrm{p}=0.168)$ were independent correlates of acute intracranial abnormalities on HCTs.

Alternatively, stratifying the cohort using the five indication groups listed above demonstrated association of both FND (OR 6.2, 95\% CI 1.2 to 33.5), and trauma, fall or syncope (OR 6.7, 95\% CI 1.9 to 23.9) with acute intracranial abnormalities on HCT, but not headache (demonstrated complete separation) or other indications $(\mathrm{p}=0.056)$.

Age, sex, race, MELD score, haemoglobin, platelet count, cirrhosis aetiology, history of peripheral artery disease (PAD), cerebrovascular accident (CVA)/transient ischaemic attack (TIA) and anticoagulant and $\mathrm{P}_{2} \mathrm{Y}_{12}$ inhibitor use prior to admission were not associated with
HCT abnormalities. Tables 3 and 4 summarise univariate and multivariate analyses, respectively.

We then sought to evaluate the number needed to screen (NNS) for a diagnosis of an acute intracranial abnormality on HCT classified by indication. The

Table 4 Multivariate analysis of correlates of acute intracranial abnormalities on head CT

\begin{tabular}{lll}
\hline Multivariate analysis & aOR $(\mathbf{9 5 \%} \mathbf{C l})$ & P value \\
\hline Aspirin use & $4.6(1.1$ to 19.2$)$ & 0.034 \\
$\begin{array}{l}\text { Presence of high-risk } \\
\text { indication }\end{array}$ & $7.3(2.3$ to 23.8$)$ & 0.001 \\
Coronary artery disease & $1.8(0.5$ to 6.6$)$ & 0.367 \\
Atrial fibrillation & $2.5(0.7$ to 9.0$)$ & 0.168
\end{tabular}

aOR, adjusted OR. 
NNS was about six times higher for low-risk indications $(\mathrm{NNS}=82)$ vs high-risk indications $(\mathrm{NNS}=14)$. Additionally, the NNS was 8 for presence of FNDs, and 13 when presenting with a fall, trauma or syncope.

For the detection of intracranial bleeding (ICH) (defined as subdural haemorrhage or haemorrhagic stroke), the NNS was almost ten times higher for lowrisk indications $(\mathrm{NNS}=136)$ vs high-risk indications $(\mathrm{NNS}=14)$. The NNS for intracranial bleeding was also 13 when presenting with a fall, trauma or syncope, and 8 for presence of FNDs.

\section{DISCUSSION}

HCTs are frequently obtained to detect acute intracranial pathology in cirrhotic patients presenting with altered mentation given this population's perceived higher bleeding risks. ${ }^{56}$ The results of this study suggest a low prevalence of acute intracranial abnormalities on HCTs in cirrhotic patients presenting with HE (2.5\%) and a low yield of routine HCTs in this population in the absence of FNDs, a history of trauma or aspirin use.

Only five $(0.96 \%)$ patients in our cohort with low-risk indications had abnormal imaging, of which only three $(0.58 \%)$ developed ICH. Additionally, in two of these patients the imaging abnormalities were thought to be incidental and not the aetiology of HE on neurosurgical evaluation. These two patients had a $5 \mathrm{~mm}$ haemorrhagic metastatic lesion and an $8 \mathrm{~mm}$ cortical haemorrhage, both without mass effect or surrounding oedema. Similarly, Kumar et al evaluated 147 HCTs in cirrhotic patients presenting with HE, in which $4 \%$ had abnormal HCT findings ${ }^{6}$ and only one $(0.7 \%)$ patient had abnormal imaging in the setting of a normal neurological exam. However, the patient did have a history of remote fall. ${ }^{6}$ All patients in Rahimi and Rockey's cohort evaluating cirrhotic patients admitted to the hospital with altered mentation found to have abnormal imaging had FNDs. ${ }^{7}$ Lastly, Donovan et al examined a cohort of patients with cirrhosis presenting with $\mathrm{HE}$ and reported higher rates of detecting ICH in patients presenting with trauma, FNDs or a history of ICH compared with patients presenting with AMS, headache or fever. ${ }^{5}$ Only one $(0.34 \%)$ of 293 patients presenting with AMS was found to have ICH. ${ }^{5}$ The three studies and our cohort support the hypothesis that routine HCTs in cirrhotic patients with HE in the absence of FNDs or history of trauma are of low diagnostic yield.

To control for potential confounders, we examined the association of antiplatelet or anticoagulant use, and history of CAD, PAD, CVA/TIAs or AF with abnormal imaging findings. Antiplatelet or anticoagulant agents may increase bleeding tendency in cirrhotic patients ${ }^{10}$ while the latter comorbidities are risk factors for developing strokes. Interestingly, aspirin but not $\mathrm{P}_{2} \mathrm{Y}_{12}$ inhibitor or anticoagulant use was found to be a positive correlate of abnormal imaging findings in our cohort even while controlling for statistically significant secondary prevention indications. However, the low event rate and lack of evaluation for metabolic comorbidities as other confounders limit the clinical utility of this observation.

The safety of low-dose aspirin in cirrhotic patients has been studied, however the data is limited and conflicting. In our cohort, aspirin use was significantly associated with a greater likelihood of presence of intracranial abnormalities on HCT. Grønbaek et al demonstrated that cirrhotic patients were five times more likely to be at risk for ICH compared with non-cirrhotic patients, and were 1.7 times more likely to have ICH if using platelet inhibitors. ${ }^{8}$ On the contrary, Patelet al reported worsening thrombocytopenia at 6-month follow-up with aspirin use however it was not associated with gastrointestinal bleeding. ${ }^{11}$ Osuga and Yoshiyasu also demonstrated that low-dose aspirin was not associated with haemorrhagic complications which included oesophageal varices. ${ }^{12}$ Lastly, Chen et al examined 1180 patients with liver cirrhosis that suffered an ischaemic stroke and were started on antiplatelet therapy at least 2 years afterwards; data showed that aspirin and clopidogrel use were not associated with increased risk of upper gastrointestinal bleeding, and that antiplatelet therapy offers decreased risk of death and readmission rates. ${ }^{13}$ Given the limited and conflicting data that is available, clinicians should weigh the risks and benefits prior to initiation of aspirin in a patient with cirrhosis, also taking into consideration whether it is for primary versus secondary CAD event prevention.

The reported study suggests an improved diagnostic yield of HCT in cirrhotic patients presenting with HE when FNDs or trauma is present $(\mathrm{NNS}=8$ and 13 , respectively). These factors could be rapidly evaluated by providers at the bedside and help triage imaging use. The importance of using imaging in higher pretest probability clinical situations was also highlighted by Donovan et al where NNS of 9 and 20 were reported for FNDs and trauma. ${ }^{5}$

HCTs are unlikely to show acute pathology in the setting of AMS alone and may contribute to increased healthcare costs. Cirrhotic patients with HE commonly have recurrent episodes requiring frequent hospitalisations. Stepanova et al evaluated the economic burden associated with HE from 2005 to 2009 and estimated each hospitalisation to cost an average of US\$46 663-US\$63 $108 .{ }^{14}$ The average cost of an HCT is variable per institution, country and insurance coverage. A study in 2015 evaluating the cost of HCTs found that, at upper-tier academic hospitals, the average cost was US $\$ 1390 \pm \mathrm{US} \$ 686 .{ }^{15}$ In the setting of known exorbitant healthcare costs in patients with recurrent HE, healthcare providers must be judicious when ordering imaging studies and should take into consideration the diagnostic yield. An initial examination including a detailed physical examination and laboratory testing to evaluate for bleeding, electrolyte abnormalities, or infection may eliminate the need to order an unnecessary HCT. As suggested earlier, reserving head imaging to higher risk patients presenting with FNDs, or a history 
of trauma, fall or syncope improves diagnostic yield and may help reduce healthcare costs.

This study carries several limitations. First, the retrospective nature of the cohort may introduce confounding bias and recall bias by providers in classifying indications for HCTs. Additionally, the setting of the study (a tertiary liver transplant centre) may not be generalisable to other community hospitals. The smaller sample size and the low event rate limits the conclusions regarding important correlates with abnormal head imaging and the ability to perform meaningful cost-effectiveness analyses. The presence of a large proportion of patients with cryptogenic cirrhosis may limit conclusions on the effect of cirrhosis aetiologies on event occurrence. Additionally, the lower MELD scores of the included population may affect generalisability of these results to patients with higher (>25) MELD scores. Finally, but importantly, an element of selection bias and under-reporting of acute intracranial events could have resulted from the use of HE ICD codes to identify the study sample rather than reviewing all hospital encounters for patients with cirrhosis in the entire study period.

In conclusion, routine HCTs in patients with cirrhosis presenting with HE with AMS in the absence of history of trauma or FNDs are of low diagnostic yield and unlikely to change patient management. Future directions may include combining data from multiple institutions to improve the sample size, racial diversity and hence the generalisability of the results. Other directions may include creation of a prospectively validated tool to triage need for HCT in cirrhotic patients presenting with HE. Examining the effects of antiplatelet and anticoagulant use on abnormal imaging findings in cirrhotic patients presenting with $\mathrm{HE}$ is certainly of interest to identify important predictors to triage imaging use in these patients.

Contributors AHan, IG, ZI and AHal were involved with the development and implementation of the study design and methods and revised the manuscript. All other authors were involved with manuscript preparation, multiple draft revisions, conception of tables and have reviewed and approved the final version of the manuscript for submission. AHan, IG, and ZI had full access to all the data in the study and take responsibility for the integrity of the data and the accuracy of the data analysis.

Funding The authors have not declared a specific grant for this research from any funding agency in the public, commercial or not-for-profit sectors.

Competing interests None declared.

Patient consent for publication Not required.

Ethics approval IRB approval obtained from the Beaumont Institutional Review Board as an exempt approval.
Provenance and peer review Not commissioned; externally peer reviewed.

Data availability statement Data are available on reasonable request. Manuscript related data are available on request from the authors and on approval of the institutional review board at Beaumont Hospital-Royal Oak of a data sharing agreement involving both parties.

Open access This is an open access article distributed in accordance with the Creative Commons Attribution Non Commercial (CC BY-NC 4.0) license, which permits others to distribute, remix, adapt, build upon this work non-commercially, and license their derivative works on different terms, provided the original work is properly cited, appropriate credit is given, any changes made indicated, and the use is non-commercial. See: http://creativecommons.org/licenses/by-nc/4.0/.

ORCID iD

Zaid Imam http://orcid.org/0000-0002-3030-6188

\section{REFERENCES}

1 Romero-Gómez M, Boza F, García-Valdecasas MS, et al. Subclinical hepatic encephalopathy predicts the development of overt hepatic encephalopathy. Am J Gastroenterol 2001;96:2718-23.

2 Weissenborn K. Hepatic encephalopathy: definition, clinical grading and diagnostic principles. Drugs 2019;79:5-9.

3 Elwir S, Rahimi RS. Hepatic encephalopathy: an update on the pathophysiology and therapeutic options. J Clin Transl Hepatol 2017;5:1-10.

4 Vilstrup H, Amodio P, Bajaj J, et al. Hepatic encephalopathy in chronic liver disease: 2014 practice guideline by the American association for the study of liver diseases and the European association for the study of the liver. Hepatology 2014;60:715-35.

5 Donovan LM, Kress WL, Strnad LC, et al. Low likelihood of intracranial hemorrhage in patients with cirrhosis and altered mental status. Clin Gastroenterol Hepatol 2015;13:165-9.

6 Kumar S, Modi R, Bhandari BM, et al. A head CT is unnecessary in the initial evaluation of a cirrhotic patient with recurrent hepatic encephalopathy. Ann Hepatol 2018;17:810-4.

7 Rahimi RS, Rockey DC. Overuse of head computed tomography in cirrhosis with altered mental status. Am J Med Sci 2016;351:459-66.

8 Grønbaek H, Johnsen SP, Jepsen P, et al. Liver cirrhosis, other liver diseases, and risk of hospitalisation for intracerebral haemorrhage: a Danish population-based case-control study. BMC Gastroenterol 2008;8:16

9 European Association for the Study of the Liver. Electronic address: easloffice@easloffice.eu, European Association for the Study of the Liver. EASL clinical practice guidelines for the management of patients with decompensated cirrhosis. J Hepatol 2018;69:406-60.

10 VCC W, Chen SW, Chou AH. Dual antiplatelet therapy in patients with cirrhosis and acute myocardial infarction - a 13-year nationwide cohort study. PLoS One 2019;14.

11 Patel SS, Guzman LA, Lin F-P, et al. Utilization of aspirin and statin in management of coronary artery disease in patients with cirrhosis undergoing liver transplant evaluation. Liver Transp/ 2018;24:872-80.

12 Osuga T, Yoshiyasu KYS, et al. Effects of low-dose aspirin administration on cirrhosis-related thrombocytopenia: report of 26 cases. Int J Clin Exp Med 2017;10:15376-83.

13 Chen C-Y, Lee K-T, Lee CT-C, et al. Effectiveness and safety of antiplatelet therapy in stroke recurrence prevention in patients with liver cirrhosis: a 2-year follow-up study. Pharmacoepidemiol Drug Saf 2012;21:1334-43.

14 Stepanova M, Mishra A, Venkatesan C, et al. In-Hospital mortality and economic burden associated with hepatic encephalopathy in the United States from 2005 to 2009. Clin Gastroenterol Hepatol 2012;10:1034-41.

15 Paul AB, Oklu R, Saini S, et al. How much is that head CT? price transparency and variability in radiology. J Am Coll Radiol 2015;12:453-7. 